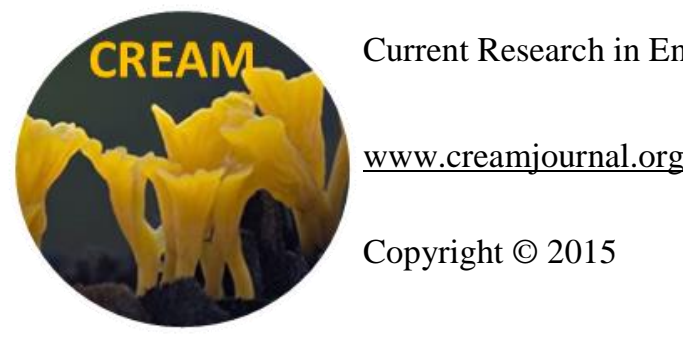

ISSN 2229-2225

Article

CREAM

Doi 10.5943/cream/5/4/7

Online Edition

\title{
A checklist of Lichens of Rajasthan, India
}

\section{Sinha GP*, Gupta P, Kar R and Joseph S}

Botanical Survey of India, Central Regional Centre, Allahabad-211002, Uttar Pradesh, India. Email: drgpsinha@gmail.com

Sinha GP, Gupta P, Kar R, Joseph S 2015 - A checklist of Lichens of Rajasthan, India. Current Research in Environmental \& Applied Mycology 5(4), 367-375, Doi 10.5943/cream/5/4/7

\begin{abstract}
The paper presents an updated list of 90 species of lichens from the state of Rajasthan, India. Three species marked by an asterisk (*) viz. Malmidea psychotrioides (Kalb \& Lücking) Kalb, Rivas Plata \& Lumbsch, Staurothele rugulosa (A. Massal.) Arnold and Willeya diffractella (Nyl.) Müll. Arg. are new records for India while 35 species marked by double asterisks (**) are new records for Rajasthan.
\end{abstract}

Key Words - Ascomycetes - new records - taxonomy - Willeya

\section{Introduction}

Rajasthan, with a geographical area of about $3,42,274 \mathrm{sq} \mathrm{km}$ is situated between $23^{\circ} 3^{\prime}-30^{\circ} 12^{\prime}$ North latitudes and $69^{\circ} 30^{\prime}-78^{\circ} 17^{\prime}$ East longitudes. Lichenologically, the state is known by the Mount Abu area of Aravali hills (Awasthi \& Singh, 1979) and some sporadic revisionary publications from the area. However, other areas of Aravali hill as well as drier areas of the state remained unexplored. To bridge this gap, the studies on lichens of Rajasthan were initiated in the year 2012 at Botanical Survey of India, Allahabad. Based on exploration and identification of new collections from various areas of the state as well as earlier information (Awasthi \& Singh, 1979, Singh \& Sinha, 2010) an updated checklist of 90 species of lichens is presented herewith (Table -1). Prior to this study, 52 species were known from the state. Out of these, three species dealt briefly, in order to facilitate their identification, marked by an asterisk (*) are new records for India and 35 species marked by double asterisks (**) are additions to the lichen biota of the state of Rajasthan.

\section{Materials \& Methods}

Specimens were collected from twelve localities of the state. The specimens were studied with standardized techniques and identified with the help of authenticated specimens available in the herbarium and published literature (Awasthi \& Singh 1979, Upreti \& Büdel 1990, Awasthi 1991, 2007, Thomson 1991, Divakar \& Upreti 2005, Gueidan \& al. 2014). The morphology and anatomy of specimens were studied under the stereomicroscope Motic SMZ-168 and compound microscope Nikon Eclipse 50i respectively. The specimens are deposited in the cryptogam herbarium section of Botanical Survey of India, Allahabad (BSA). 


\section{Results}

Malmidea psychotrioides (Kalb \& Lücking) Kalb, Rivas Plata \& Lumbsch in Kalb, Rivas Plata, Lücking \& Lumbsch, Biblioth. Lichenol. 106: 165. 2011.

Fig 1

Thallus corticolous, crustose, rimose, sub-verrucose, greyish-white; prothallus greenish-black; medulla white, $\mathrm{K}-$. Apothecia rounded, sessile, 0.5-2.2 $\mathrm{mm}$ diam.; disc plane, brown to dark brown; margin thick, smooth, white, prominent; excipulum paraplectenchymatous, brown, encrusted with hydrophobic granules, olive-grey, $\mathrm{K}+$ green, 20-40 $\mu \mathrm{m}$ wide; epihymenium reddish-brown, 15-18 $\mu \mathrm{m}$ thick; hymenium colourless, 64-70 $\mu \mathrm{m}$ high; hypothecium brown, 40-70 $\mu \mathrm{m}$ thick, $\mathrm{K}-$; paraphyses simple, capitate, apices brown; asci clavate, 8-spored, 65-80 $\times 8-12 \mu \mathrm{m}$, I+ blue; ascospores colourless, simple, ellipsoid, thick walled, halonate, 13-15(-18) $\times(6-) 7-8 \mu \mathrm{m}$, perispore $1.03 \mu \mathrm{m}$ thick. Thallus $\mathrm{K}+$ yellow; medulla $\mathrm{K}-, \mathrm{C}-, \mathrm{KC}-, \mathrm{P}-$; atranorin present.

The species, previously known from Brazil, was collected from bark of a tree in an open place along road side forest.

Specimen examined - India, Rajasthan, Mount Abu, on way between Gurusikhar-Trevor's Tank, $24^{\circ} 38^{\prime} 36.9^{\prime \prime}$ N, $72^{\circ} 46^{\prime} 40^{\prime \prime}$ E, alt. 1540 m, 07. 09. 2012, G.P. Sinha \& R. Kar 7853.

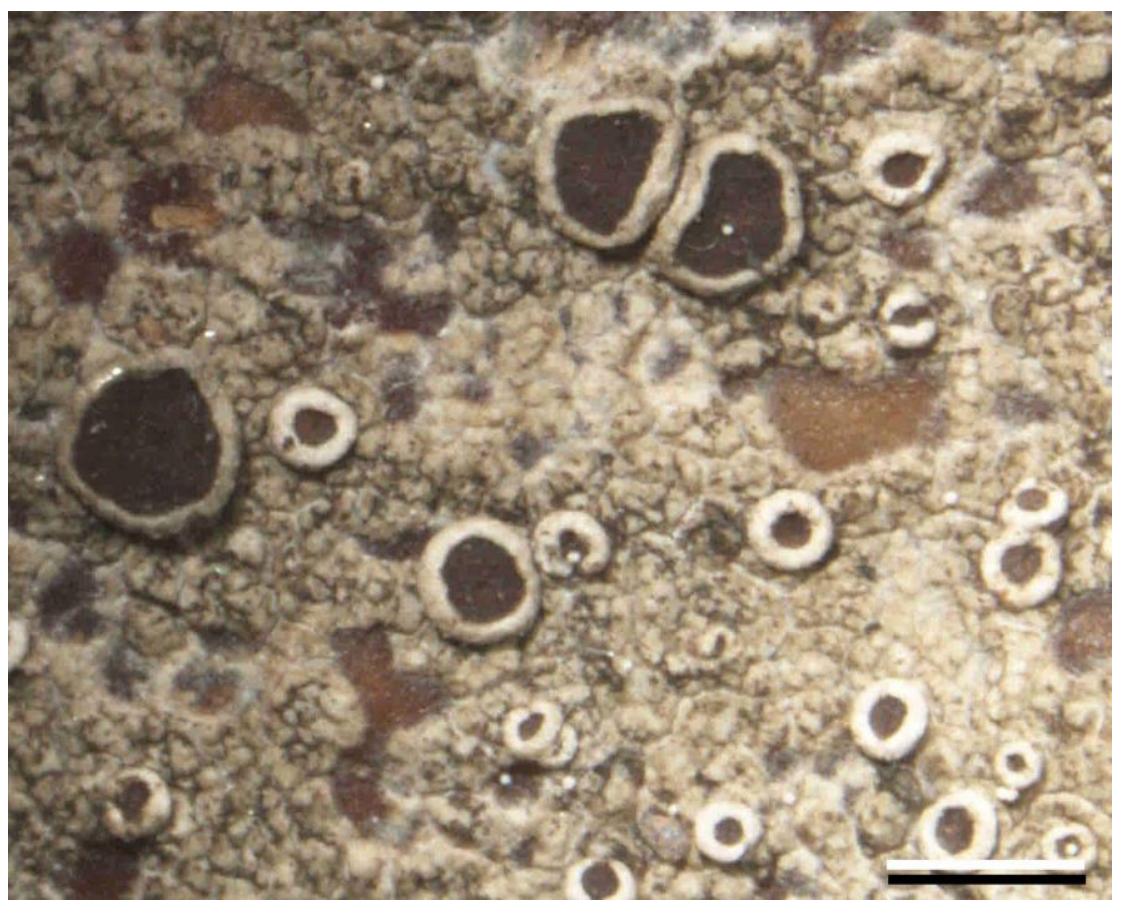

Fig. 1 - Habit - Malmidea psychotrioides (Kalb \& Lücking) Kalb, Rivas Plata \& Lumbsch - Bar $=2.5 \mathrm{~mm}$

Staurothele rugulosa (A. Massal.) Arnold, Verh. K. K. Zool.-Bot. Ges. Wien 47: 389. 1897. Polyblastia rugulosa A. Massal., Mem. Lichenogr.: 139. 1853.

Thallus saxicolous, crustose, rimose-areolate, areoles with deep cracks, flat to slightly concave, 0.2-0.7 mm diam., whitish-grey; medulla white; prothallus absent. Perithecia black, embedded in the thalline areoles; peridium brownish, 37-52 $\mu \mathrm{m}$ wide, with brown involucrellum around the ostiole, dimidiate at base; centrum colourless, 98-148 $\mu \mathrm{m}$ high, I+ yellow turning red, with rounded algal cells, 2.21-3.83 $\mu \mathrm{m}$ diam.; paraphyses absent, short periphysoids present; asci 4-spored; ascospores colourless, narrowly ellipsoid-ovoid, muriform, $(23-) 29-35(-42) \times(10-) 11-12(-16) \mu \mathrm{m}$. Thallus and medulla $\mathrm{K}-, \mathrm{C}-, \mathrm{KC}-, \mathrm{P}-$.

The pantropical species was collected from calcareous rocks in an open scrub forest.

Specimen examined - India, Rajasthan, Sajjangarh Wildife Sanctury, $24^{\circ} 35^{\prime} 38.5^{\prime \prime} \mathrm{N}, 73^{\circ} 38^{\prime}$ 22.4" E, alt. 912 m, 18.09.2012, G.P. Sinha \& R. Kar 7971. 


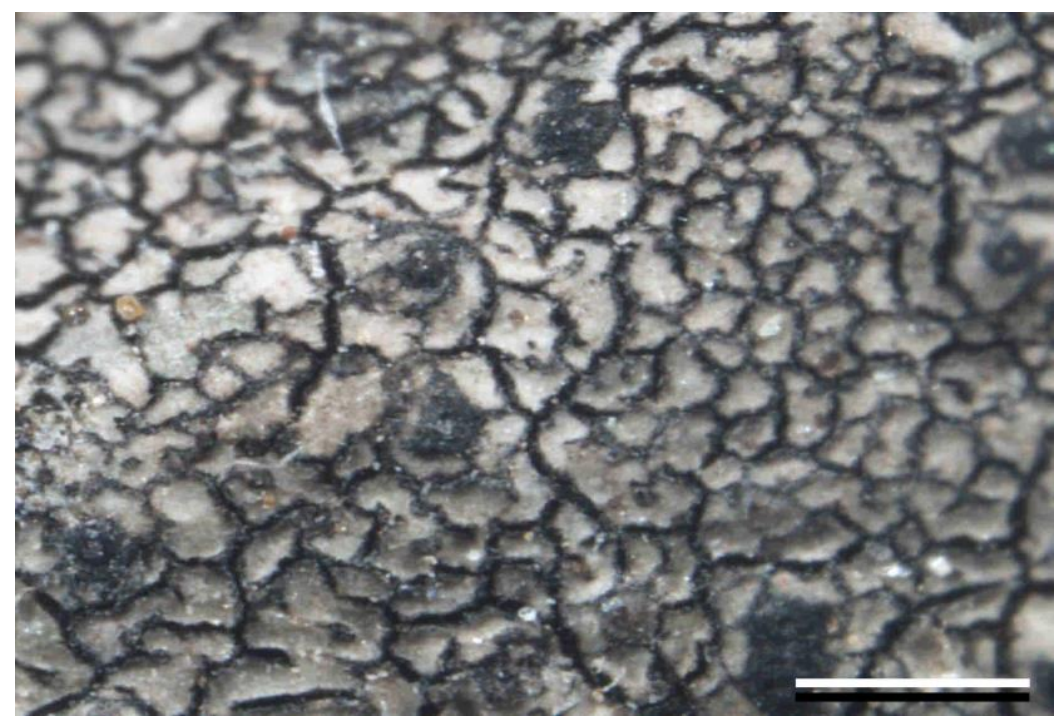

Fig. 2 - Habit - Staurothele rugulosa (A. Massal.) Arnold - Bar $=2.5 \mathrm{~mm}$

Willeya diffractella (Nyl.) Müll. Arg., Flora 66: 345. 1883. Verrucaria diffractella Nyl., Mém. Soc. Acad. Maine Loire 4: 33. 1858. Staurothele diffractella (Nyl.) Tuck., Gen. Lich.: 258. 1872. $\quad$ Fig 3

Thallus saxicolous, crustose-subsquamulose, rimose-areolate, areoles with deep cracks, slightly convex, 0.3-0.9 mm diam., yellowish-grey, brown with green appearance; medulla white; prothallus absent. Perithecia black, embedded in the thalline areoles; ostiole raised and prominent; peridium dark brownish, with a dark brown involucrellum around the ostiole; involucrellum dimidiate at base; centrum colourless, I+ yellow turning red, with rounded algal cells, 2.70-3.65 $\mu \mathrm{m}$ diam.; paraphyses absent, short pariphysoids present; asci 4-8 sporeed; ascospores colourless, ellipsoid-ovoid, muriform, $(16-) 17-19(-25) \times(7-) 8-9(-12) \mu \mathrm{m}$. Thallus and medulla $\mathrm{K}-, \mathrm{C}-, \mathrm{KC}-, \mathrm{P}-$.

The pantropical species was collected from calcareous rocks in an open place along road side forest.

Specimen examined - India, Rajasthan, Sawaimadhopur, Kusalidra forest, on way between Pali and Sawaimadhopur, $25^{\circ} 56^{\prime}$ 08.4" N, 76 $22^{\prime}$ 46.4" E, alt. 252 m, 30.09.2013, G.P. Sinha \& R. Kar 8517.

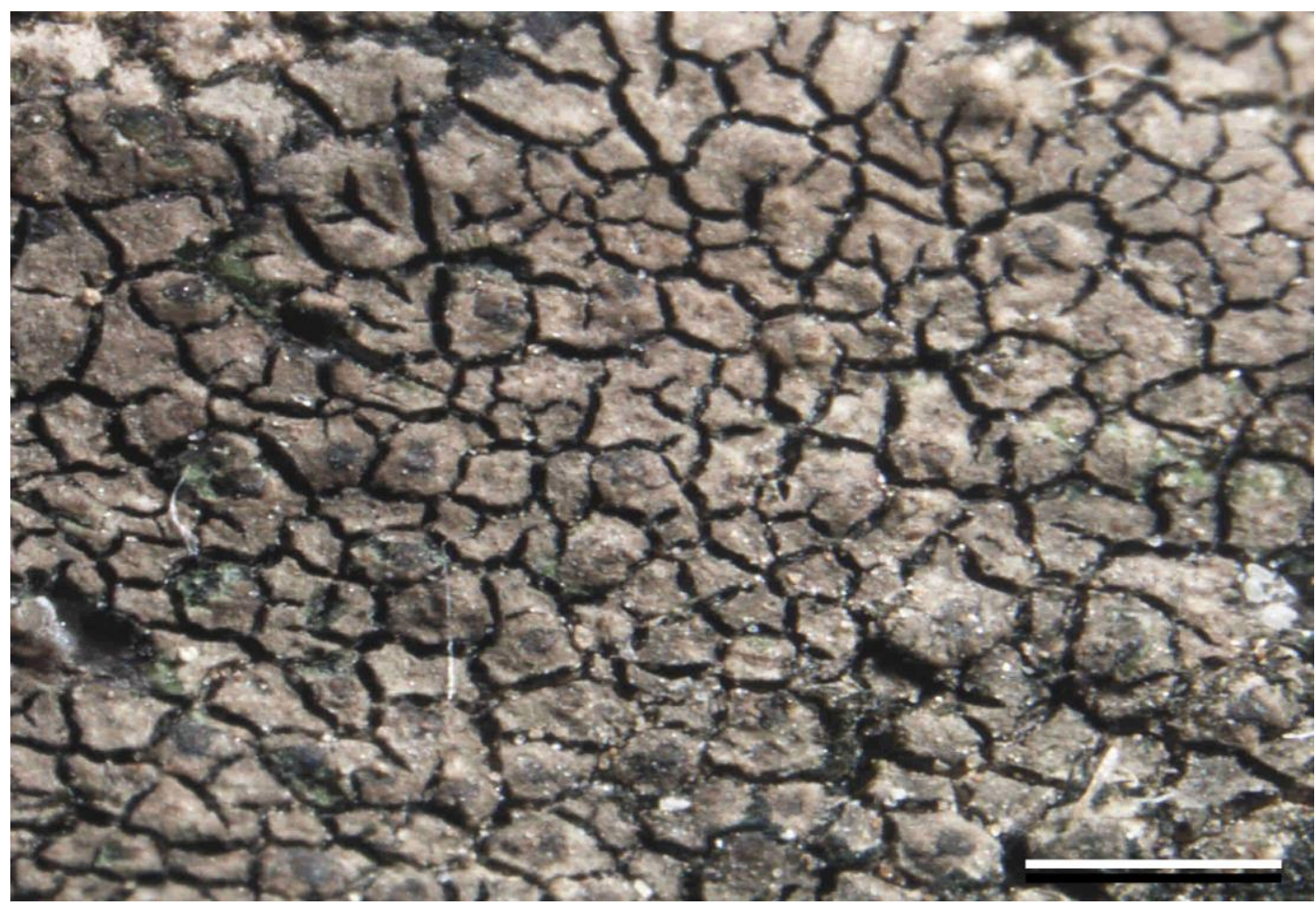

Fig 3 - Habit - Willeya diffractella (Nyl.) Müll. Arg. - Bar = $1.0 \mathrm{~mm}$ 
Table 1 Enumeration of lichens of Rajasthan (1. Ajmer 2. Banswara 3. Baran 4. Bharatpur 5. Bhilwara 6. Bundi 7. Chittougarh 8. Kota 9. Rajsamand 10. Sawaimadhopur 11. Sirohi ( Mt. Abu) 12. Udaipur

$\mathrm{Co}$ - Corticolous, $\mathrm{Cr}$ - Crustose, Fo - Foliose, Sa - Saxicolous, Sq - Squamulose, subfr - Subfruticose, $(*)-$ New records for India, $(* *)-$ New records for Rajasthan)

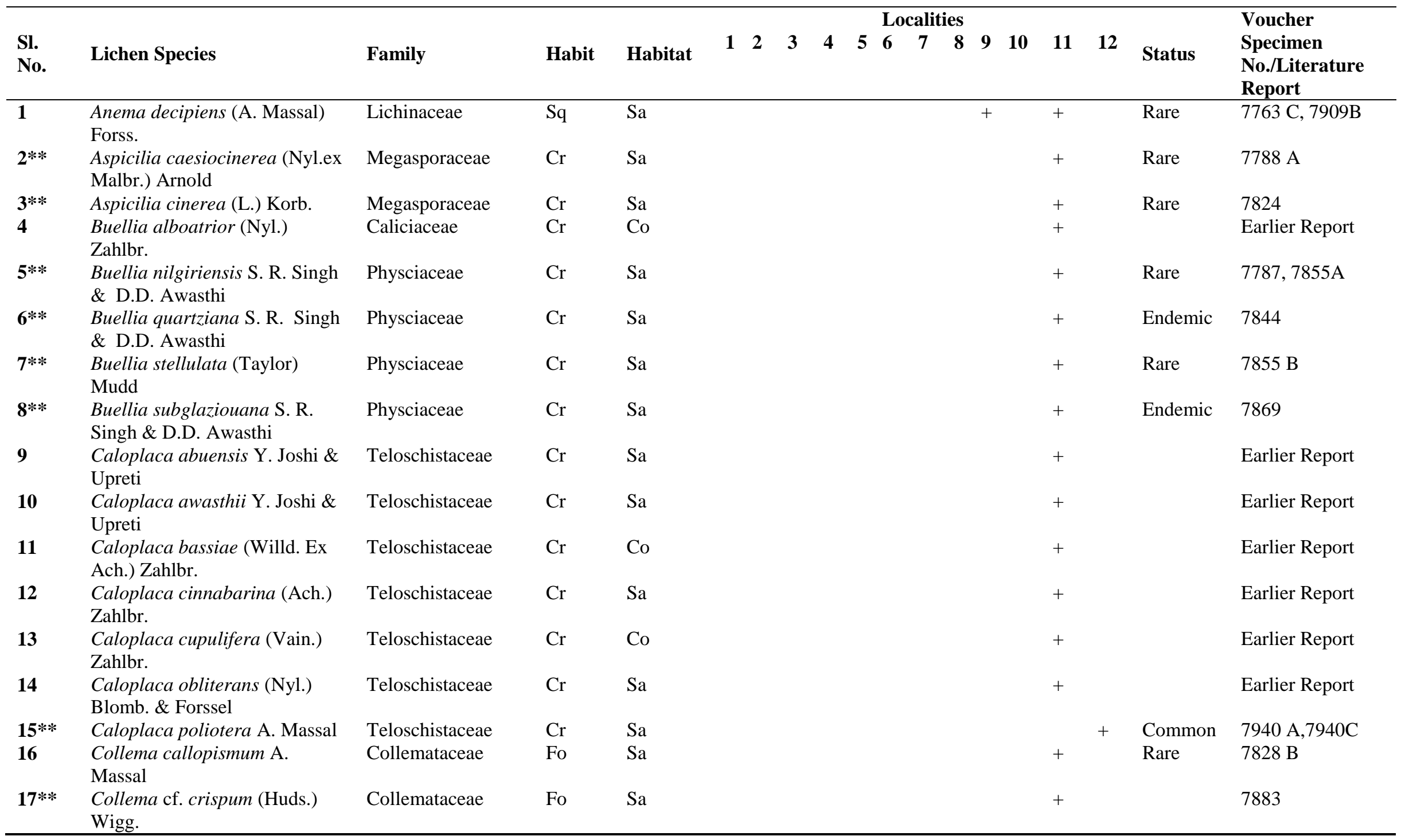




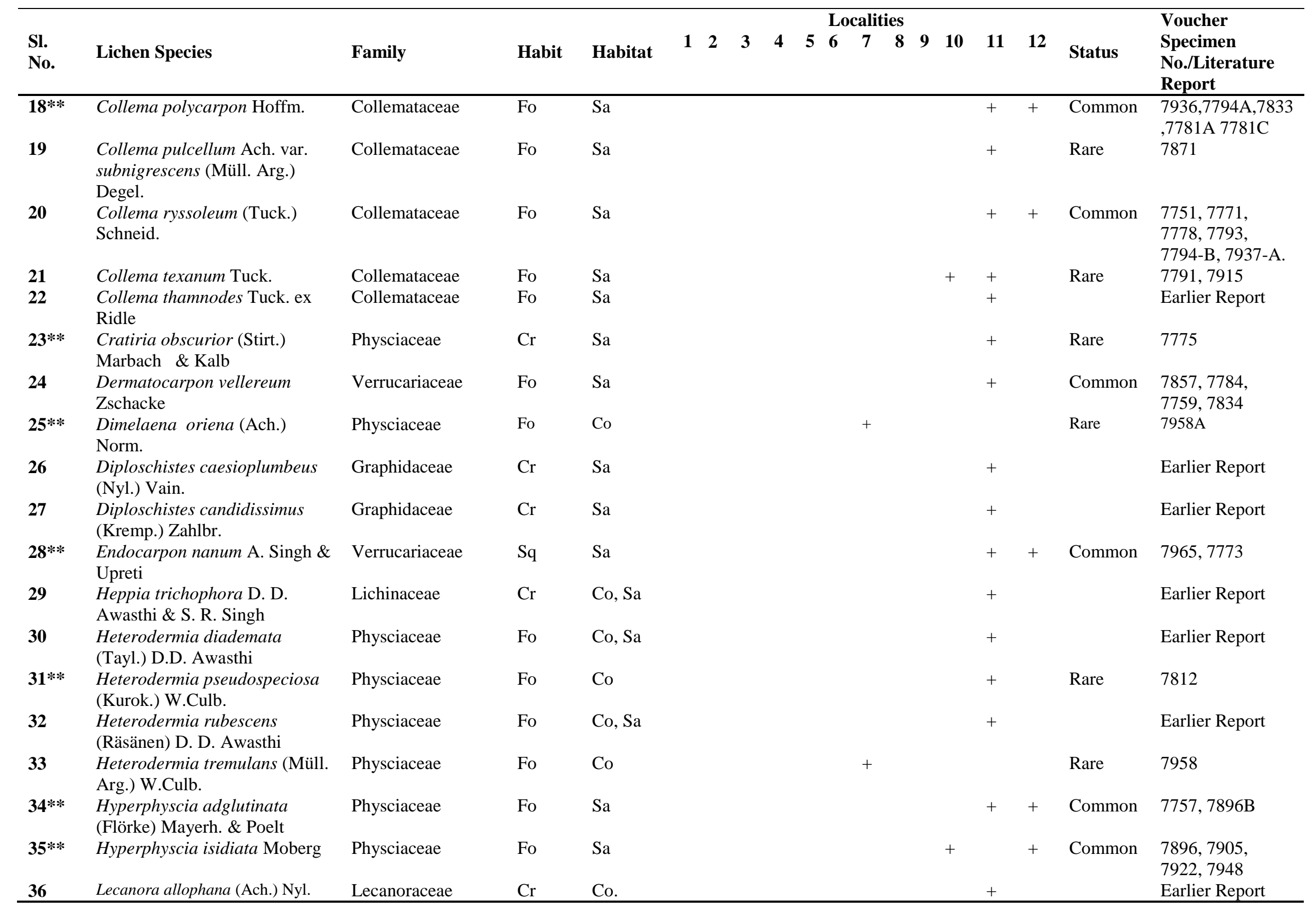




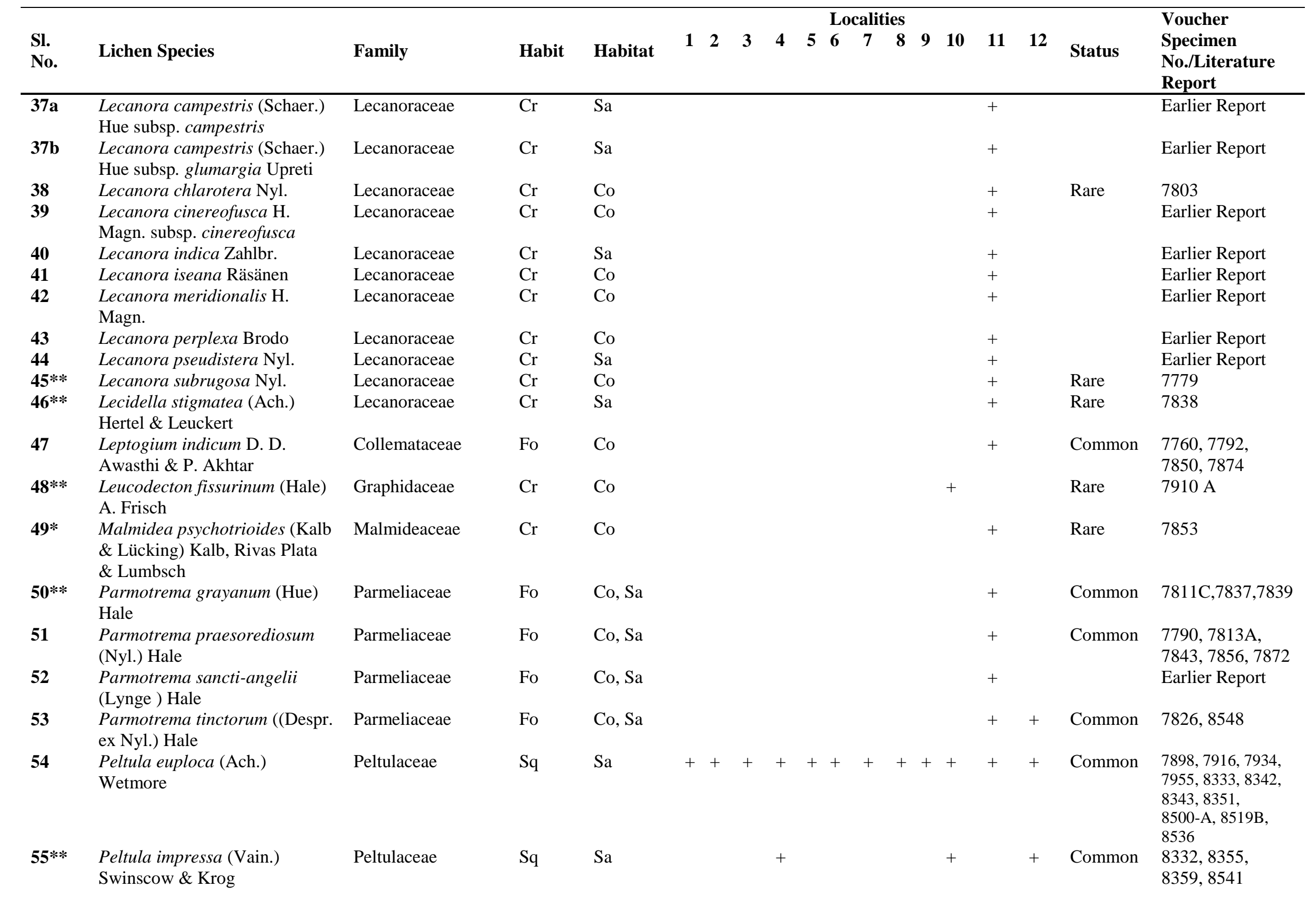




\begin{tabular}{|c|c|c|c|c|c|c|c|c|c|c|c|c|c|c|c|c|c|c|}
\hline \multirow[b]{2}{*}{$\begin{array}{l}\text { Sl. } \\
\text { No. }\end{array}$} & \multirow[b]{2}{*}{ Lichen Species } & \multirow[b]{2}{*}{ Family } & \multirow[b]{2}{*}{ Habit } & \multirow[b]{2}{*}{ Habitat } & \multirow[b]{2}{*}{1} & \multirow[b]{2}{*}{2} & \multirow[b]{2}{*}{3} & \multirow[b]{2}{*}{4} & \multicolumn{5}{|c|}{ Localities } & \multirow[b]{2}{*}{10} & \multirow[b]{2}{*}{11} & \multirow[b]{2}{*}{12} & \multirow[b]{2}{*}{ Status } & \multirow{2}{*}{$\begin{array}{l}\text { Voucher } \\
\text { Specimen } \\
\text { No./Literature } \\
\text { Report } \\
\end{array}$} \\
\hline & & & & & & & & & 5 & 6 & 7 & 8 & 9 & & & & & \\
\hline $56 * *$ & $\begin{array}{l}\text { Peltula obscurans var. } \\
\text { desertifolia (Zahlbr.) Wetmore }\end{array}$ & Peltulaceae & $\mathrm{Sq}$ & $\mathrm{Sa}$ & & & + & & & & & & & & & & & 8498B \\
\hline $57 * *$ & $\begin{array}{l}\text { Peltula patellata (Bagl.) } \\
\text { Swinscow \& Krog }\end{array}$ & Peltulaceae & $\mathrm{Sq}$ & $\mathrm{Sa}$ & & & & + & & & + & & & + & & & Common & $\begin{array}{l}7961,8330 \\
8362,8364 \mathrm{~B}\end{array}$ \\
\hline $58 * *$ & $\begin{array}{l}\text { Peltula placodizans (Zahlbr.) } \\
\text { Wetmore }\end{array}$ & Peltulaceae & $\mathrm{Sq}$ & $\mathrm{Sa}$ & & & & + & & & & & & + & & & Common & $\begin{array}{l}8331,8350 \\
8367\end{array}$ \\
\hline $59 * *$ & $\begin{array}{l}\text { Peltula tortusa }(\text { Nees }) \\
\text { Wetmore }\end{array}$ & Peltulaceae & $\mathrm{Sq}$ & $\mathrm{Sa}$ & & & & & & & & & + & & & & Rare & 7911,7912 \\
\hline $60 * *$ & $\begin{array}{l}\text { Peltula zahlbruckneri (Hausse) } \\
\text { Wetmore }\end{array}$ & Peltulaceae & $\mathrm{Sq}$ & $\mathrm{Sa}$ & + & + & + & + & + & + & + & + & + & + & + & + & Common & $\begin{array}{l}7879,7890, \\
7956,8336, \\
8344,8353, \\
8356 \mathrm{~A}, 8365, \\
8498 \mathrm{C}, 8500 \mathrm{~B}\end{array}$ \\
\hline 61 & Pertusaria cf. laetea (L.) Arn. & Pertusariaceae & $\mathrm{Cr}$ & Co & & & & & & & & & & & + & & & Earlier Report \\
\hline $62 * *$ & $\begin{array}{l}\text { Pertusaria leucoplaca Müll. } \\
\text { Arg. }\end{array}$ & Pertusariaceae & $\mathrm{Cr}$ & $\mathrm{Sa}$ & & & & & & & & & & & & + & Common & 7953,7946 \\
\hline 63 & $\begin{array}{l}\text { Pertusaria leucostoma (Bernh.) } \\
\text { A. Massal. }\end{array}$ & Pertusariaceae & $\mathrm{Cr}$ & Co & & & & & & & & & & & + & & & Earlier Report \\
\hline 64 & Pertusaria pertusa (L.) Tuck. & Pertusariaceae & $\mathrm{Cr}$ & $\mathrm{Sa}$ & & + & & & & & & & & & + & + & Common & $\begin{array}{l}8553,7768,7867, \\
7758,7761\end{array}$ \\
\hline 65 & $\begin{array}{l}\text { Phaeophyscia ciliata (Hoffm.) } \\
\text { Moberg }\end{array}$ & Physciaceae & Fo & $\mathrm{Sa}$ & & & & & & & & & & & + & & Common & $\begin{array}{l}7823,7841 \\
7852 \mathrm{~A}\end{array}$ \\
\hline 66 & $\begin{array}{l}\text { Phaeophyscia hispidula (Ach.) } \\
\text { D.D.Awasthi \& S.R. Singh var. } \\
\text { hispidula }\end{array}$ & Physciaceae & Fo & $\mathrm{Sa}$ & & & & & & & & & & & + & & Common & 7757B \\
\hline $67 * *$ & $\begin{array}{l}\text { Phaeophyscia hispidula (Ach.) } \\
\text { D.D.Awasthi \& S.R. Singh var. } \\
\text { exornatula (Zahlbr.) Moberg }\end{array}$ & Physciaceae & Fo & $\mathrm{Sa}$ & & & & & & & & & & & + & & Common & $\begin{array}{l}7811 \mathrm{~A}, 7842 \mathrm{~B} \\
7860\end{array}$ \\
\hline 68 & $\begin{array}{l}\text { Phoeophyscia nigricans } \\
\text { (Floerke) Moberg }\end{array}$ & Physciaceae & Fo & $\mathrm{Sa}$ & & & & & & & & & & & + & & Rare & 7799 \\
\hline $69 * *$ & $\begin{array}{l}\text { Phaeophyscia orbicularis } \\
\text { (Neck.) Moberg }\end{array}$ & Physciaceae & Fo & $\mathrm{Sa}$ & & + & & & & & & & & & + & & Common & $\begin{array}{l}7756,7800 \\
7822,7840 \mathrm{~B} \\
7852 \mathrm{~B}, 7875 \\
7899,8556\end{array}$ \\
\hline 70 & $\begin{array}{l}\text { Phylliscum abuense D.D. } \\
\text { Awasthi \& S.R. Singh }\end{array}$ & Lichinaceae & $\mathrm{Sq}$ & $\mathrm{Sa}$ & & & & & & & & & & & + & & Rare & 7859 \\
\hline 71 & $\begin{array}{l}\text { Physcia abuensis D.D. Awasthi } \\
\text { \& S.R. Singh }\end{array}$ & Physciaceae & Fo & Co & & & & & & & & & & & + & & Rare & $\begin{array}{l}7754,7810 \\
7820\end{array}$ \\
\hline
\end{tabular}




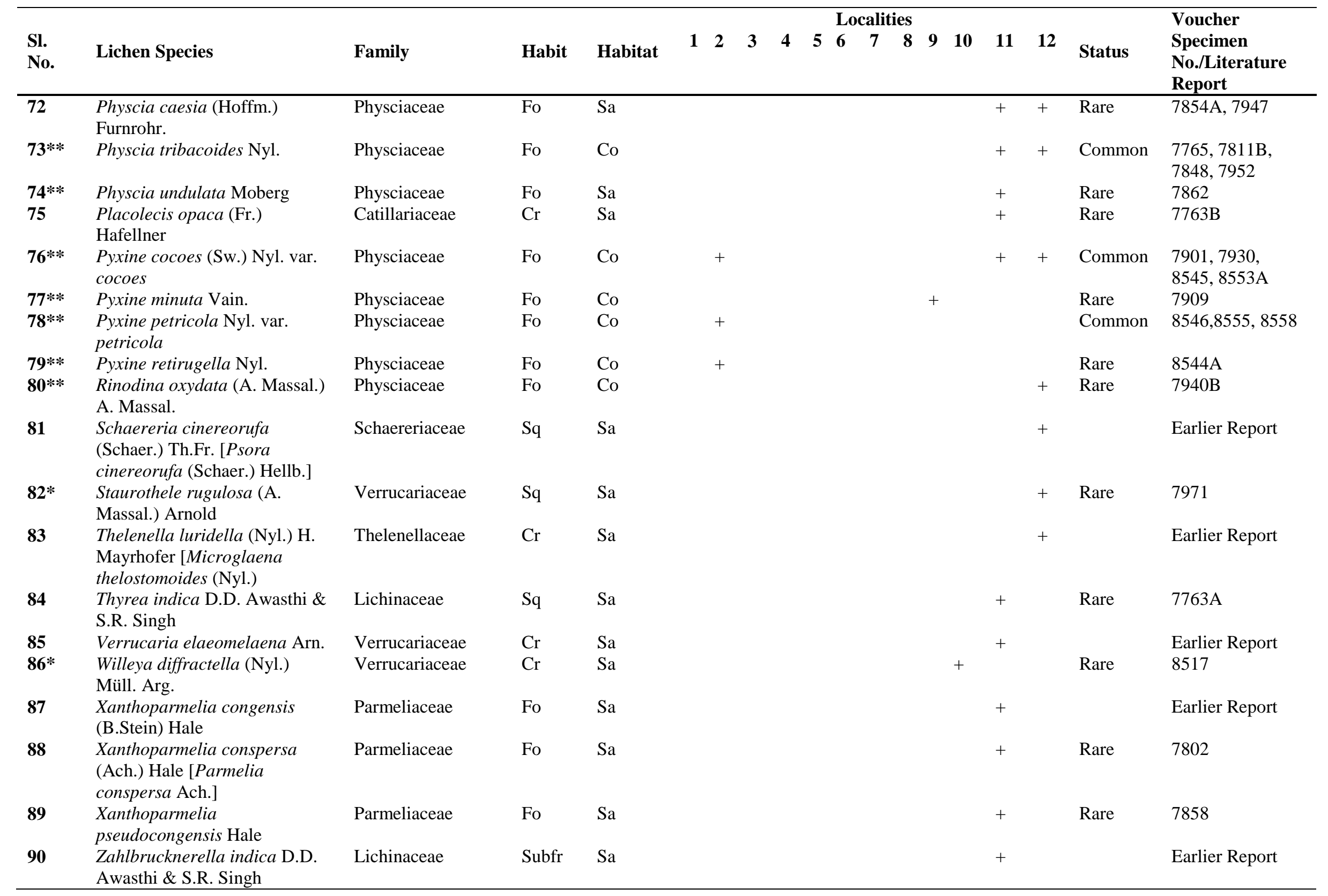




\section{Acknowledgements}

The authors are thankful to Director, Botanical Survey of India, Kolkata for facilities and to authorities of Forest department, Govt. of Rajasthan for providing logistic support in protected areas.

\section{References}

Awasthi DD. 1991 - A Key to the Microlichens of India, Nepal and Sri Lanka. Biblioth. Lichenol. 40, $1-337$.

Awasthi DD. 2007 - A Compendium of the Macrolichens from India, Nepal and Sri Lanka. Bishen Singh Mahendra Pal Singh, Dehra Dun, India.

Awasthi DD, Singh SR. 1979 - New or otherwise interesting lichens from Mt. Abu,Rajasthan, India. Norwej. J. Bot. 26, 91-97.

Divakar PK, Upreti DK. 2005 - Parmeloid Lichens in India (A Revisionary Study). Bishen Singh Mahendra Pal Singh, Dehra Dun, India.

Gueidan C, Van Do T, Lu Thi N. 2014 - Phylogeny and taxonomy of Staurothele (Verrucariaceae, lichenized ascomycetes) from the karst of northern Vietnam. Lichenologist 46(4), 515-533.

Singh KP \& Sinha GP. 2010 - Indian Lichens: An Annotated Checklist. Botanical Survey of India, Kolkata.

Thomson JW. 1991 - The lichen genus Staurothele in North America. Bryologist 94, 351-367.

Upreti DK, Büdel B. 1990 - The lichen genera Heppia and Peltula in India. J. Hattori Bot. Lab. No. 68, 279-284. 